\title{
Pénurie de médecins: la solution vient-elle des femmes?
}

\author{
La pénurie de médecins se fait de plus en plus ressentir en Suisse. Si au début du \\ siècle on parlait encore d'un afflux de médecins, force est de constater que la \\ tendance s'est clairement inversée depuis. Malgré la reconnaissance de $\mathbf{2 1 0}$ diplômes \\ étrangers [1], de nombreux postes sont en effet restés vacants en 2010. Cette situa- \\ tion peut en partie être expliquée par la part de femmes chez les étudiants et le \\ nombre croissant de femmes médecins qui obtiennent leur diplôme.
}

\section{Esther Bieri}

Esther Bieri de Lucerne est superviseuse et coach HES. Elle travaille en tant que cheffe de projet dans des hôpitaux suisses.

* Nom modifié par la rédaction

Correspondance:

FMH Communication Jacqueline Wettstein

Elfenstrasse 18

CH-3000 Berne 15

Tél. 0313591111

Fax 0313591112

kommunikation(at)fmh.ch
Pendant de nombreuses années, les horaires de travail étaient adaptés en fonction des besoins de la clinique et n'étaient pas plafonnés. En 2005, une limite de 50 heures par semaine a été introduite dans les hôpitaux pour les médecins-assistants et pour une partie des chefs de clinique.

En parallèle, les attentes de la population envers la médecine ne cessent de s'accroître, et de nombreux postes ont donc dû être créés pour faire face à ces nouveaux défis. Or on constate qu'un nombre important de postes d'assistants et de cadres restent vacants, et dans certaines disciplines, dont la médecine de famille, la relève fait d'ores et déjà défaut.

L'attrait pour les études de médecine reste cependant élevé, comme en témoigne la demande de places d'étude qui ne semble pas faiblir: ce ne sont en effet pas moins de 2936 personnes qui se sont inscrites en

\section{La FMH soutient des projets concrets}

Pour la Fédération des médecins suisses (FMH), la pénurie de médecins serait notamment due au fait que l'on ne forme pas suffisamment de médecins. Elle a d'ailleurs consacré un chapitre de ses «Objectifs stratégiques pour la période législative 20082012» au soutien à la relève professionnelle et s'engage à différents niveaux politiques pour une augmentation de $20 \%$ du nombre d'étudiants en médecine.

Une deuxième cause à l'origine de cette pénurie est la difficulté rencontrée par les jeunes médecins pour concilier vie de famille et carrière. En effet, beaucoup interrompent leur carrière ou y mettent carrément un terme au moment de fonder une famille. Forte de ces constatations, la FMH soutient des projets concrets élaborés par ses membres et qui visent à instaurer des conditions de travail adaptées aux exigences actuelles et favorables à la famille (crèches, travail à temps partiel, etc.) dans les institutions du secteur de la santé. médecine humaine auprès des universités de Bâle, Berne, Fribourg et Zurich pour l'année académique 2011/2012, soit 3,5 fois plus d'inscriptions que de places disponibles. Partant de ce constat, la Fédération des médecins suisses (FMH) propose d'augmenter de $20 \%$ le nombre de places d'étude.

\section{$63 \%$ d'étudiantes}

Si l'on regarde de plus près les statistiques estudiantines de l'Université de Zurich, la féminisation de l'effectif des étudiants en médecine humaine est une évidence. En l'an 2000, la part d'étudiantes se montait déjà à $63 \%$. Mais les femmes ont-elles les mêmes opportunités de carrière que leurs homologues masculins? Peut-on concilier une carrière de médecin avec une vie de famille? Une étude de l'hôpital universitaire de Zurich portant sur l'égalité des chances des futurs médecins hommes et femmes [2] montre clairement toute l'ambiguïté de la situation: si la part de femmes se monte à $40 \%$ parmi les médecins-assistants, et qu'elle est encore de $30 \%$ parmi les chefs de clinique, plus on monte dans les échelons hiérarchiques et plus les femmes se font rares: elles ne sont en effet plus que $8 \%$ parmi les médecins dirigeants.

\section{L'époux endosse le rôle de père au foyer}

Le Dr Karin Alpiger* est mère de deux enfants en âge scolaire. Elle a terminé ses études de médecine en 1993 avec l'objectif de faire carrière dans la chirurgie. Durant sa formation postgradué - lors de laquelle elle a accompli les années de formation et le nombre d'opérations requises pour obtenir le titre de spécialiste FMH en chirurgie -, cette jeune médecin talentueuse a bénéficié d'un important soutien de la part de ses chefs. "Mais plus on avance dans sa carrière, plus ce soutien diminue», remarque-t-elle. Au lieu de cela, elle s'est vue attribuer toujours plus de tâches ingrates, qui empiétaient sur sa formation. «Mes deux enfants sont nés alors que j'occupais un poste de cheffe de clinique. Mes grossesses ont été sans com- 
plication ce qui m'a permis de continuer à travailler à plein temps jusqu'à terme. Heureusement, mon mari a eu le courage d'endosser le rôle de père au foyer. Comme il avait déjà fait carrière en tant que directeur informatique dans une grande maison d'édition, il a souhaité m'aider afin que je puisse me concentrer sur ma carrière à l'hôpital.»

\section{Temps partiel pour les médecins-cadres: la solution?}

Pour K. Alpiger, il n'a jamais été question de travailler à temps partiel: «La chirurgie est un métier manuel qui nécessite beaucoup d'entraînement et d'expérience. A temps partiel, cela m'aurait pris beaucoup plus de temps pour devenir cheffe de clinique», explique-t-elle. Maintenant qu'elle a son propre cabinet, elle peut plus facilement aménager ses horaires en fonction de sa vie de famille, mais continue malgré tout à travailler à plein temps: «Après une formation aussi longue, j'ai acquis pas mal de savoir-faire et d'expérience. Et j'ai décidé d'en faire profiter mes patients.» Elle comprend cependant le désir de jeunes médecins qui souhaitent combiner carrière et famille en travaillant à temps partiel: «Vu la pénurie de médecins, il faudrait prendre en compte la perte de savoir-faire qui se profile et prendre des mesures concernant le taux d'occupation.»

\section{Crèches ouvertes jusqu'à $\mathbf{2 2 h}$}

A l'heure actuelle, les cliniques qui proposent des postes à temps partiel aux chirurgiens occupant un poste de médecin-cadre sont peu nombreuses. L'une d'entre elles est la clinique orthopédique de l'hôpital cantonal de Lucerne à Wolhusen. A sa tête, deux femmes et un homme qui se partagent deux postes de chefs de clinique. Tous trois ont une famille. «J'ai vu le talent de ces futurs médecins et j'ai souhaité continuer à les soutenir - qu'ils soient hommes ou femmes», raconte le Dr Richard F. Herzog, médecin-chef. «Oui, c'est risqué. Cela ne fonctionne que si les chefs de clinique à temps partiel sont prêts à respecter scrupuleu- sement leurs horaires de travail, comme tous les autres collègues d'ailleurs.» L'effectif du personnel et l'organisation de la clinique ne permettent pas aux médecins de partir à 18h. Les médecins-cadres assument l'entière responsabilité envers leurs patients et doivent donc rester jusqu'à ce que le dernier patient ait été examiné et le dernier rapport d'opération dicté. «Il est urgent que les hôpitaux mettent sur pied des crèches ouvertes de $6 \mathrm{~h}$ à $22 \mathrm{~h}$ et faciles d'accès pour les parents.» Contrairement à K. Alpiger, le Dr Herzog pense que le temps partiel a un rôle important à jouer pour endiguer durablement la pénurie de médecins. Pour lui, c'est la seule solution pour maintenir durablement dans le secteur de la santé les mères qui ont obtenu un titre de spécialiste en chirurgie. «Mais pour cela, il faut absolument adapter les conditions de travail, comme la possibilité de faire garder ses enfants par des tiers ou de se spécialiser dans un sousdomaine.»

\section{Coordination de la formation jusqu'au titre de spécialiste}

Aussi bien K. Alpiger que R. F. Herzog souhaitent que la FMH et les sociétés de discipline médicale soutiennent la création de régions de formation, composées de différents grands hôpitaux, pour permettre aux candidates et candidats de planifier leur formation jusqu'à l'obtention du titre de spécialiste. Car la formation de candidates à temps partiel est plutôt compliquée à gérer pour les responsables de clinique; planifier sur le long terme est essentiel pour les hôpitaux afin d'éviter le manque de personnel et de pallier la pénurie croissante de spécialistes.

\section{Références}

1 Suter P, de Haller J, Täuber M, Gassmann B. Les besoins futurs en médecins en Suisse. Bull Méd Suisses. 2006;87(17):713-4.

2 Jacquemart C, Boos L. Gleichberechtigte Nachwuchsförderung von Ärztinnen und Ärzten am Universitätsspital Zürich; 2000.

\section{Trois femmes, trois comptes rendus}

Barbara Buddeberg-Fischer, Professeur et médecin spécialiste en psychiatrie et psychothérapie $\mathrm{FMH}$, en psychiatrie et psychothérapie d'enfants et d'adolescents FMH ainsi qu'une formation complémentaire en médecine psychosomatique et psychosociale (ASMPP)

Arrivant bientôt au terme de mon cursus professionnel, je me demande, lorsque j'y repense, comment je suis parvenue à tout concilier: l'acquisition de deux titres de spécialiste, mon activité en cabinet et, paral- lèlement, l'éducation de mes enfants et mon activité scientifique. Très tôt, je me suis constitué une seconde branche d'activité dans la recherche en sciences sociales, en menant par exemple des études sur les troubles de l'alimentation chez les adolescents. En tant que chercheuse, je trouve dommage que la science reste un secteur très masculin dans lequel les femmes se font toujours plus rares. 12\% des hommes optent en effet pour une carrière médicale universitaire contre seulement $2 \%$ des femmes. 
De même, les femmes restent sous-représentées dans les étages supérieurs des hôpitaux. J'espère que cette situation évoluera dans les prochaines années puisque les femmes représentent depuis quelques années la majorité des diplômés en médecine. Les jeunes médecins femmes et hommes ne sont plus disposés à travailler 24 h sur 24 et c'est là, selon moi, un point positif. Malheureusement, la formation postgraduée et les conditions d'engagement s'axent trop souvent encore sur les carrières masculines. La semaine de 50 heures dans la phase de formation postgraduée est loin d'être respectée dans de nombreux hôpitaux et disciplines médicales. Souvent, les crèches des hôpitaux ne répondent pas non plus aux besoins des médecins femmes, par exemple en termes de temps de travail. Cela doit absolument changer. Il faut beaucoup plus d'emplois à temps partiel de 80 à $60 \%$ pour les femmes afin qu'elles puissent concilier famille et travail. Selon moi, la formation postgraduée peut être accomplie avec un taux d'activité de $80 \%$, même en chirurgie. Comme on le sait, la qualité d'un médecin ne dépend pas du nombre de ses heures supplémentaires, mais entre autres de sa personnalité.

Je conseille aux étudiantes en médecine et à leurs collègues de décider le plus tôt possible du titre de spécialiste qu'elles souhaitent acquérir. Une décision précoce permet en effet d'aménager la formation postgraduée de manière optimale et efficace. Il serait par exemple envisageable de centraliser la formation postgraduée: un an avant l'examen d'Etat, les étudiants en médecine optent pour une discipline médicale et s'inscrivent à l'instance centrale correspondante.

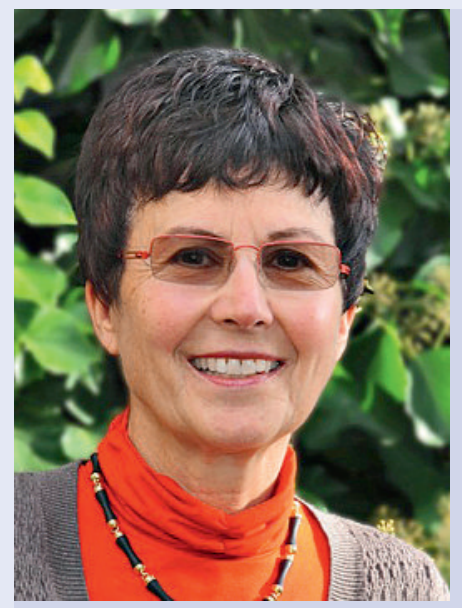

Barbara Buddeberg-Fischer: «Aujourd'hui, les femmes représentent la majorité des diplômés en médecine. II s'avère donc nécessaire de procéder à des changements dans la formation postgraduée, dans les conditionscadres institutionnelles des hôpitaux, dans le monde professionnel et également dans la recherche.»

Celle-ci leur soumet alors diverses offres pour l'ensemble de la durée de la formation postgraduée auxquelles ils peuvent postuler. Je trouve que la formation postgraduée médicale dure actuellement trop longtemps. On pourrait la limiter à quatre ou cinq ans sans perte de qualité et définir les objectifs annuels à atteindre. La formation postgraduée se poursuit dans la formation continue pendant toute la durée de l'exercice de la profession. Une formation postgraduée raccourcie et efficace se révèlerait être un avantage, notamment pour les femmes dans notre profession.

\section{Dr Brigitte Muff, Médecin-cheffe en chirurgie, spécialiste FMH en chirurgie avec formation ap- profondie en chirurgie viscérale, chirurgie géné- rale et traumatologie, et attestation de formation complémentaire en médecine psychosociale et psychosomatique}

Cela fait plus de 20 ans que j'exerce en tant que chirurgienne et je suis depuis 2005 médecin-cheffe de la clinique chirurgicale de l'hôpital de Bülach. Malgré toutes ces années de pratique, j'ai toujours beaucoup de plaisir à exercer mon métier, tant sur le plan opératoire qu'au chevet des patients. La formation des jeunes médecins me tient également très à cœur: les familiariser avec le quotidien d'une clinique, leur transmettre un savoir-faire chirurgical, c'est important pour moi. Mais ce métier a aussi ses inconvénients qui ne sont pas, comme on le pense souvent, les longues heures de présence ou les services de garde astreignants, mais le fait que de plus en plus de personnes, qui n'ont rien à voir avec le monde médical, veulent nous dicter ce que nous devons faire ou ne pas faire. Dans les hôpitaux, la

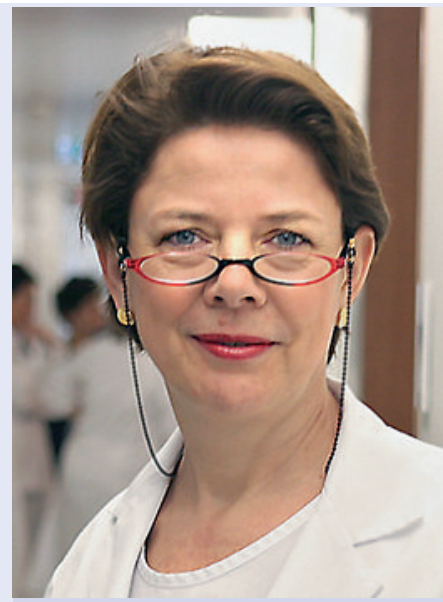

Dr med Brigitte Muff: «Malgré toutes ces années de pratique, j'ai toujours beaucoup de plaisir à exercer mon métier, tant sur le plan opératoire qu'au chevet des patients.»

charge administrative n'a cessé de croître ces dernières années. Il y a donc forcément de plus en plus 
de recoupements (inutiles) avec le corps médical. Devoir constamment justifier nos actes auprès des différents départements de l'hôpital est non seulement démoralisant et démotivant, mais cela ne favorise pas non plus le développement de la qualité. Comme les hôpitaux sont dirigés par des managers, cela entraîne automatiquement un changement de paradigme. En raison de leurs connaissances en économie d'entreprise, ceux-ci sont convaincus que les hôpitaux, en tant que prestataires de services, peuvent être gérés comme n'importe quelle entreprise de biens de consommation*. Avant, il y avait des personnes malades - autrement dit des patients - qui avaient besoin de traitements et les coûts qui en découlaient étaient compensés par des subventions, des primes, ou de manière directe. Aujourd'hui, on ne parle plus de patients mais de clients et ce n'est plus le traitement le mieux adapté à chaque individu qui est au centre des préoccupations, mais l'optimisation des gains. Il s'agit de mettre en œuvre un contrat social en y appliquant les règles de l'économie de marché - quel paradoxe!

En tant que femme et mère de deux jeunes femmes, on me demande souvent comment j'arrive à concilier travail et vie de famille. Or on ne devrait plus poser cette question aujourd'hui. Il devrait en effet aller de soi que les mères puissent aussi assumer des fonctions dirigeantes dans tous les domaines professionnels. Certaines conditions doivent bien évidemment être remplies. Il faut en premier lieu que non seulement les parents, mais aussi la société, reconnaissent qu'il n'est pas négatif de faire garder ses enfants. Il faut créer suffisamment de places dans les crèches et ce service devrait aussi être garanti pour les parents dont le père ou la mère travaille par rou- lement. Ce modèle est d'ailleurs déjà appliqué en France et dans les pays scandinaves. A l'heure actuelle, les femmes-médecins sont largement sousreprésentées dans les sphères dirigeantes des hôpitaux et dans l'enseignement. Or si la femme le veut (et c'est la condition principale) et que le monde hospitalier n'est pas d'emblée hostile aux femmes et aux mères, cette inégalité peut être corrigée. Il est également possible d'instituer un modèle de partage de poste, à l'instar de la maternité de l'hôpital Triemli de Zurich, co-dirigé avec succès par deux médecins-cheffes à temps partiel.

Je souhaite à la future génération de médecins qu'à l'avenir, les diktats étrangers à la médecine tels que la loi sur le travail pour les médecins-assistants (plutôt défavorable aux disciplines opératoires) ou les formulaires à remplir pour de soi-disant questions d'assurance-qualité ne continuent pas à proliférer. En outre, il s'agit de stopper l'économicisation croissante de la médecine de sorte que le patient soit à nouveau au centre des préoccupations et qu'il ne soit pas considéré comme un client qui achète des prestations. Quant à mon avenir personnel, je pourrais tout à fait imaginer intervenir dans une région en crise ou dans un pays en voie de développement. Dans un lieu où il s'agit justement de soigner des malades sans arrière- pensées économiques.

* Mathias Binswanger décrit très bien ce phénomène dans son livre «Sinnlose Wettbewerbe: Warum wir immer mehr Unsinn produzieren.», aux éditions Herder. Bernard Lown traite également cette question dans son livre «Die verlorene Kunst des Heilens. Anstiftung zum Umdenken», aux éditions Suhrkamp (tous deux non traduits en français).

Patrizia Kündig, étudiante en médecine à la Faculté de médecine de I'Université de Bâle

Aujourd'hui en cinquième année de médecine humaine à l'Université de Bâle, je savais déjà avant de passer ma maturité que j'allais embrasser cette filière. Et je suis encore aujourd'hui convaincue d'avoir fait le bon choix. D'une manière générale, je souhaiterais que l'on accorde une plus grande place à la pratique déjà lors de la formation universitaire. Certes, la formation pratique est plus présente qu'il y a encore quelques années, mais uniquement dans le cadre de certains cours. Les cours magistraux sont encore monnaie courante. Or, même si cette méthode d'enseignement convient à certaines branches, ce n'est pas le cas de toutes. Nous ferions bien de revoir cette didactique et d'analyser le cursus actuel pour voir quelles sont les matières qui sont, à juste titre, enseignées sous cette forme, et combien d'heures sont nécessaires pour enseigner les diffé-

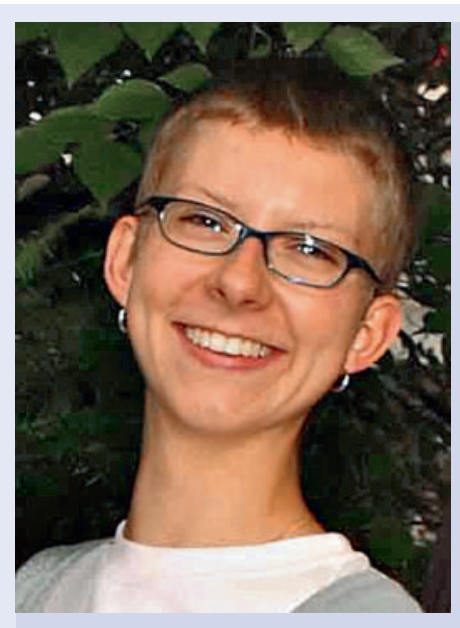

Patrizia Kündig: «ll est grand temps que les choses changent et que les conditions de travail des médecins soient adaptées aux besoins actuels.» 
rents sujets. Pour certaines branches, une méthode d'enseignement pratique ou axée sur des problèmes concrets serait bien plus indiquée. Pour mes collègues et moi, il est primordial que les objectifs d'apprentissage reflètent l'activité que nous exercerons plus tard et qu'ils tiennent compte de l'évolution de notre système de santé. Je n'ai pas encore choisi mon domaine de spécialisation, mais je m'intéresse actuellement beaucoup à la médecine d'urgence, je me vois donc bien travailler en médecine intensive ou à la REGA. Je prévois également d'acquérir mon titre de spécialiste aussi rapidement. Comme je souhaite participer à l'évolution de la formation médicale, je suis aujourd'hui déjà engagée en politique associative puisque je fais partie du comité de l'Association suisse des étudiants en médecine (swimsa).

Après mes études, je souhaite continuer à œuvrer au sein de la politique professionnelle et pour la formation des médecins. Je m'imagine également fonder une famille et j'espère que cela sera compatible avec mon activité de médecin, car je ne veux pas abandonner ma profession. Nombre d'autres étudiantes et étudiants en médecine n'ont pas l'intention de renoncer à leur carrière ou à leur vie de famille, il est donc grand temps que l'on nous donne la possibilité d'exercer notre profession à temps partiel et que l'on nous y encourage. Beaucoup de femmes médecins mais également de plus en plus d'hommes souhaitent travailler à temps partiel, sans être pour autant considérés comme des paresseux et se retrouver sur le bord de la route. Nous avons aussi besoin de plus de crèches dans les hôpitaux, et leurs offres doivent être développées et adaptées aux besoins des médecins. Par ailleurs, la nouvelle génération ne souhaite pas renoncer systématiquement au temps libre et au repos. Il est par conséquent urgent que la durée maximale légale de la semaine de travail des médecins-assistants (50 heures) soit une réalité pratique, et pas seulement théorique. Pour répondre à l'évolution actuelle - plus de femmes que d'hommes suivent des études de médecine et les besoins des futurs médecins ne sont plus les mêmes -, il est essentiel que le monde professionnel des médecins se remette en question, et en particulier que les employeurs se montrent plus flexibles. Cependant, même si les structures et les conditions de travail des autres professions sont plus confortables, je reste passionnée par la médecine, me réjouis d'exercer cette profession et espère qu'il me restera assez de temps pour développer une vraie relation avec les patients. 